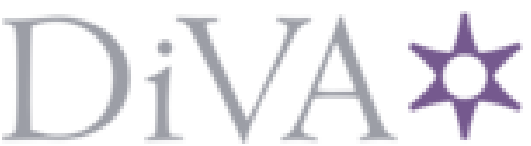

http://www.diva-portal.org

Postprint

This is the accepted version of a paper presented at 2019 21st European Conference on Power Electronics and Applications (EPE'19 ECCE Europe).

Citation for the original published paper:

Bitsi, K., Wallmark, O., Bosga, S. (2019)

Many-objective Optimization of IPM and Induction Motors for Automotive Application In:

N.B. When citing this work, cite the original published paper.

Permanent link to this version:

http://urn.kb.se/resolve?urn=urn:nbn:se:kth:diva-264770 


\title{
Many-objective Optimization of IPM and Induction Motors for Automotive Application
}

\author{
Konstantina Bitsi ${ }^{1}$, Oskar Wallmark ${ }^{1}$, and Sjoerd Bosga ${ }^{1,2}$ \\ ${ }^{1}$ KTH Royal Institute of Technology, Dept. of Electric Power \\ and Energy Systems, Stockholm, Sweden \\ ${ }^{2}$ ABB Corporate Research, Västerås, Sweden \\ E-mails: bitsi@kth.se, owa@kth.se,bosga@kth.se
}

\section{Acknowledgments}

This research project is supported in part by the Swedish Energy Agency (Energimyndigheten).

\section{Keywords}

$\ll$ Automotive application》, «Electric vehicles》, «Evolutionary many-objective optimization》, $\ll$ Finite element method $\gg, \ll$ Induction motors $\gg, \ll$ Interior-permanent magnet motors $\gg$

\begin{abstract}
This paper presents a Pareto-optimality-based optimization methodology suitable for the design of electrical motors in automotive applications. The proposed many-objective evolutionary algorithm is utilized in this study case for the optimization of an interior permanent-magnet (IPM) synchronous motor and an induction motor (IM), considering as criteria the motors' torque capability, efficiency as well as torque density. Finite-element (FE) models of the investigated motor topologies are developed and incorporated in the optimization process in order to ensure an accurate estimation of their electromagnetic performance. The attainment of the targeted specifications by the final optimal designs validates the efficacy of the implemented optimization algorithm.
\end{abstract}

\section{Introduction}

The market of electric vehicles (EVs) is constantly expanding, as the development of EVs is considered one of the most promising ways to ensure a sustainable energy future [1]. Therefore, the design of suitable electrical traction motors is of great importance, with criteria regarding efficiency, cost, torque and power density playing a determining role [2]. The permanent magnet synchronous motor is proven a strong candidate for automotive applications, as it exhibits high torque density and high efficiency [3]. In particular, the interior permanent-magnet (IPM) topology is widely adopted due to its superior flux weakening capability and the advantage of constant power over a broad speed range [4]. Nevertheless, an induction motor (IM) is still a competitive choice, as it constitutes a lower-cost, reliable and free of rare-earth-material solution [5],[6].

For the optimization process of a traction motor, the use of Pareto-optimality-based evolutionary algorithms yields a set of Pareto-optimal solutions, which represent different compromises among the considered design-objectives [7],[8]. This optimization method results in a pragmatic approach to the problem, as it offers the appropriate freedom to the researcher to arrive, after further analysis, to the selection of a single solution depending on the examined application [9]. A widely used evolutionary multi-objective algorithm is the Non-dominated Sorting Genetic Algorithm II (NSGA-II) [10]. NSGA-II is based on an elitist principle, highlights non-dominated solutions and attempts to maintain the appropriate diversity by using a crowding distance operator [11],[12]. 
In an automotive application, however, the optimization process should typically consider more than three commonly opposing objectives (i.e. torque, efficiency, power density etc). In these cases, NSGA-II can face difficulties due to the substantial increase of the non-dominated part of the population, the computationally-expensive assessment of the crowding-distance criterion and the rather unsuccessful crossover between effectively distant neighbouring members [13]. To tackle the above issues, the algorithm NSGA-III was introduced in [13]. This optimization procedure demonstrates a superior performance when dealing with many-objective optimization problems, as it further supports the diversity preservation by utilizing the concept of multiple predefined reference points.

In this paper, a many-objective optimization algorithm based on the concept of NSGA-III is presented. This algorithm is utilized in order to study and compare the resulting optimal geometries of IPM and IM designs. To faciliate this comparison, the motor from the Toyota Prius 2010 Hybrid System was considered as a reference geometry [14].

\section{Description of finite element models}

\section{Electromagnetic models}

The reference design of this study is an 8-pole, 48 stator-slot IPM motor with distributed stator winding and V-shaped permanent magnets [14]. Based on this design, the parametric finite element (FE) model of an IPM motor of similar geometrical characteristics was developed. This 2-D FE model is shown in Fig. 1a. In addition, the FE model of an IM was designed (Fig. 1b). The goal of this IM design is to achieve similar operational and geometrical characteristics as the IPM design. For this purpose, an 8-pole geometry with 48 stator slots and 64 rotor copper bars was selected. An electric circuit was added in the rotor of the IM in order to properly short-circuit the copper bars, while taking into account the end-ring resistance.

To achieve less time-consuming electromagnetic solutions, the symmetries of the motors are considered. In the case of the IPM motor, only 1/8 of the geometry (i.e., 1 pole) can be simulated in the FE model, while in the case of the IM, 1/4 of the geometry is needed (i.e., 1 pole-pair) in order to properly shortcircuit the induced currents in the electrical circuit of the rotor.

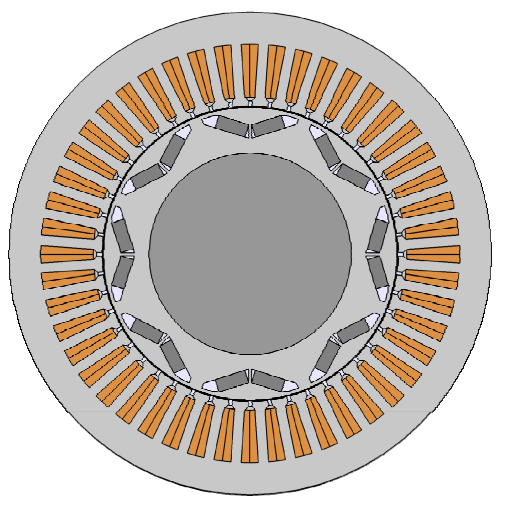

(a)

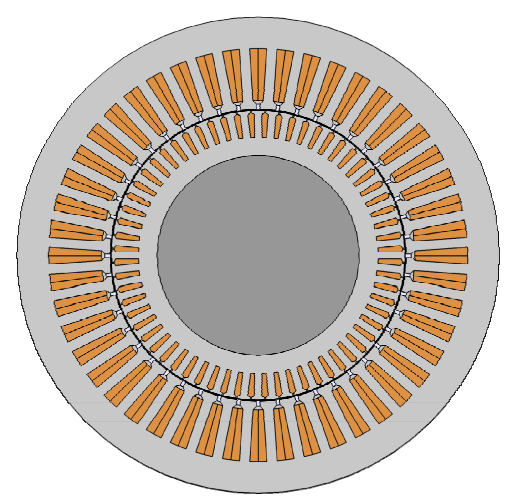

(b)

Fig. 1: (a) IPM and (b) IM FE models.

The electromagnetic analysis of all FE models was performed using COMSOL Multiphysics ${ }^{\circledR 1}$. Moreover, MATLAB ${ }^{\circledR 2}$ was used as a scripting tool for setting up and solving the COMSOL models.

\section{Target specifications}

In this paper, the aim of our analysis is to optimize and compare both motors at a selected nominal operating point. Therefore, the selected target specifications are shown in Table I. It should be noted that these values were chosen based on the performance of the reference electric motor [14].

\footnotetext{
${ }^{1}$ Comsol Multiphysics ${ }^{\circledR}$ is a registered trademark of Comsol AB, Stockholm, Sweden.

${ }^{2} \mathrm{Matlab}^{\circledR}$ is a registered trademark of The Mathworks Inc., Natick, Ma, USA.
} 
Table I: Target specifications

\begin{tabular}{l|c}
\hline Speed, $n_{\mathrm{n}}(\mathrm{rpm})$ & 2500 \\
\hline DC voltage, $V_{\mathrm{DC}}(\mathrm{V})$ & 400 \\
\hline Torque, $T_{\text {ref }}(\mathrm{Nm})$ & 140 \\
\hline Efficiency, $\eta_{\text {ref }}(\%)$ & $\max$ \\
\hline Torque density, $\tau_{\text {ref }}(\mathrm{Nm} / \mathrm{kg})$ & $\max$ \\
\hline
\end{tabular}

\section{Design parameters}

The key variables of the two parametric FE models are presented in Fig. 2. In order to enable a fair comparison between the IPM and the IM, certain design parameters were kept constant between the two motor designs during the overall analysis. The values of these design constants are listed in Table II. In addition, certain design parameters were selected to be varied during the IPM and IM optimal design

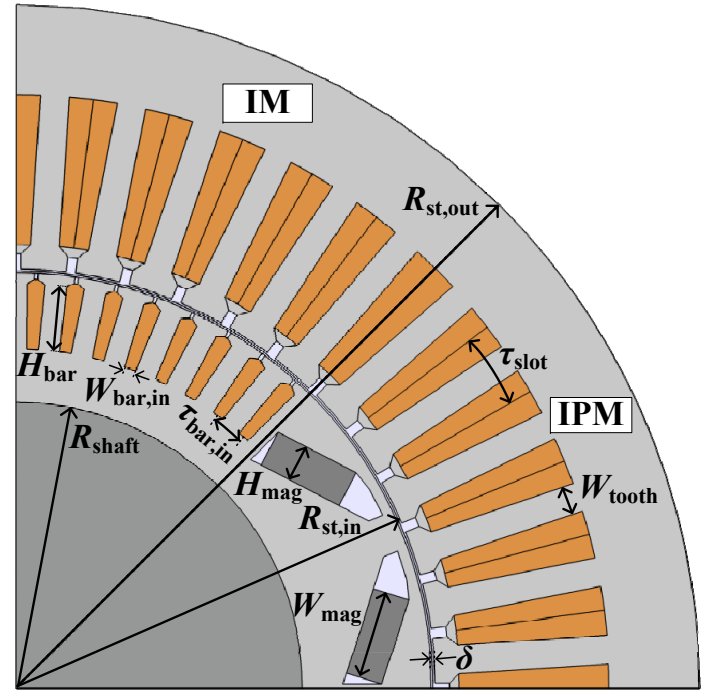

Table II: Design constants

\begin{tabular}{l|c}
\hline Stator outer radius, $R_{\text {st,out }}(\mathrm{mm})$ & 132 \\
\hline Air-gap, $\delta(\mathrm{mm})$ & 0.73 \\
\hline $\begin{array}{l}\text { Stator } \mathrm{rms} \text { conductor current } \\
\text { density, } J_{\mathrm{Cu}}\left(\mathrm{A} / \mathrm{mm}^{2}\right)\end{array}$ & 10 \\
\hline Stator-slot fill factor, $f_{\mathrm{Cu}}(-)$ & 0.45 \\
\hline
\end{tabular}

Fig. 2: Design parameters of the IM and IPM designs.

analysis (Table III). These design parameters constitute the optimization decision variables that are used as inputs in the multi-objective optimization routine described in the following section. The choice of these parameters was made based on their significant impact on the performance of the motor designs as well as the high dependency of secondary parameters on them.

\section{Evolutionary multi-objective optimization of IPMs and IMs}

\section{Implemented optimization algorithm}

Evolutionary optimization algorithms are widely used optimization techniques, which use stochastic operators in a procedure inspired by processes occurring in the biological evolution [9]. These robust optimizing methods employ a population approach, in which the solution of every iteration (i.e., generation) consists of multiple individuals and evolves to a new selection of population members in the next iteration. Their algorithm is defined by a set of determined steps: selection, crossover and mutation of the population. Their utility is based on the simplicity of their structure, the non-use of derivatives in the performed cost calculations as well as their ability to identify and converge to the global minima of the investigated problem.

The evolutionary multi-objective optimization problems are characterised by a number of objectives to be minimized. Similar to the case of the mono-objective problems, their optimization algorithm tries to 
Table III: Key design variables

\begin{tabular}{c|l|c}
\hline Design & Parameter name and unit & Expression \\
\hline \hline IPM & Split ratio (-) & $\chi_{\text {split }}=\frac{R_{\text {st,in }}}{R_{\text {st,out }}}$ \\
\hline & Stator tooth-width ratio (-) & $\chi_{\text {tooth }}=\frac{W_{\text {tooth }}}{\tau_{\text {slot }}}$ \\
\hline & Magnet width (mm) & $W_{\text {mag }}$ \\
\hline & Magnet height (mm) & $H_{\text {mag }}$ \\
\hline \hline & Aspect ratio (-) & $\chi_{\text {aspect }}=\frac{L_{\text {active }}}{2 \cdot R_{\text {stout }}}$ \\
\hline IM & Split ratio (-) & $\chi_{\text {split }}=\frac{R_{\text {st,in }}}{R_{\text {st,out }}}$ \\
\hline & Stator tooth-width ratio (-) & $\chi_{\text {tooth }}=\frac{W_{\text {tooth }}}{\tau_{\text {slot }}}$ \\
\hline & Rotor bar-width ratio (-) & $\chi_{\mathrm{w}, \text { bar }}=\frac{W_{\text {bar,in }}}{\tau_{\text {bar,in }}}$ \\
\hline & Rotor bar-height ratio (-) & $\chi_{\mathrm{h}, \text { bar }}=\frac{H_{\text {bar }}}{R_{\text {rot,out }}-R_{\text {shaft }}}$ \\
\hline & Aspect ratio (-) & $\chi_{\text {aspect }}=\frac{L_{\text {active }}}{2 \cdot R_{\text {st,out }}}$ \\
\hline
\end{tabular}

identify appropriate values of the decision variables that minimize each of the objective functions within pre-set boundaries. The outcome of the search is a set of Pareto-optimal solutions.

The algorithm presented on this paper is based on the framework of NSGA-III [13]. Following the concept of NSGA-II [10], NSGA-III includes the principle of elitism among the individuals, which involves the inclusion of the fittest members of each generation to the next one unchanged. In addition, it puts emphasis on the preservation of suitable non-dominated solutions between successive generations in order to maintain the appropriate diversity in the population and, thus, escape local minima. The novelty of NSGA-III compared to NSGA-II lies in its ability to handle successfully many-objective optimization problems (i.e., having more than three objectives), as it introduces the use of multiple predefined reference points in order to further support the diversity preservation [13]. Thereupon, solutions associated to every reference point can be emphasized to obtain sets of Pareto-optimal solutions spread over a large domain.

The structure of the implemented algorithm for the optimization of the IPM and IM motor models is presented in Fig. 3. As a first step, an initial population, $P_{1}$, of size $N$ is created randomly by varying the decision variables within their defined boundaries. For each member of the population, its objective functions are evaluated using a different cost-evaluation procedure based on which motor type is investigated (either IM or IPM). These cost-evaluation procedures are described in detail in following subsections.

Next, in each generation $i$, additional individuals (offspring) are created though the processes of crossover $\left(Q_{\mathrm{cr}, \mathrm{i}}\right)$ and mutation $\left(Q_{\text {mut,i }}\right)$ of existing population members. In the crossover stage, a predefined number of individuals (depending on the crossover probability, $p_{c}$ ) is selected randomly from the population and paired up. For every pair of individuals, two additional offspring are created by combining half the decision variables of one individual-parent with half the variables of the other. In the mutation stage, a number of decision variables (based on the mutation probability, $p_{m}$ ) of randomly selected individuals are changed according to a mutation step-size, $\sigma_{m}$, which expresses how far the mutated variable can be from the original value. These processes help the population to converge in optimal solutions faster, while maintaining the appropriate diversity to escape local minima.

As a next step, the union of the current population $\left(P_{i}\right)$ and the generated offspring $\left(Q_{\mathrm{cr}, \mathrm{i}}\right.$ and $\left.Q_{\mathrm{mut}, \mathrm{i}}\right)$ is normalized and sorted in fronts using the concept of Pareto non-domination. All the population members, starting from the first front, are added to the population of the next generation, $P_{i+1}$, until the front where the population size exceeds $N$. Then, the final members of the population, $K$, are selected from the last front based on the niche counting of the reference points [13]. These reference points are uniformly defined on a normalized hyper-plane, which is equally inclined to all objective axes and has a single 


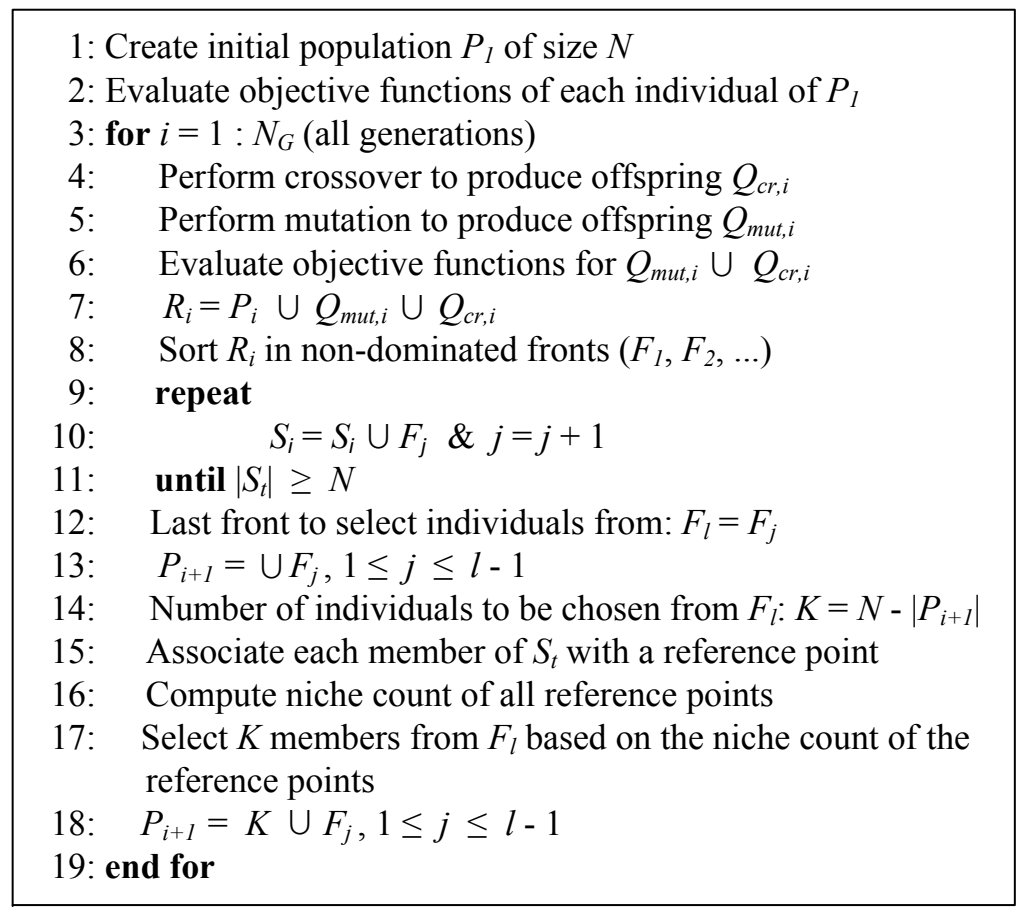

Fig. 3: Structure of the implemented evolutionary algorithm.

intercept with each axis. Each population member can be associated with a reference point, based on the shortest perpendicular distance of the member from the reference lines (lines connecting the reference points with the origin). Thereafter, the remaining members $K$ are selected by starting from the reference point with the least associated members in the new population pool in order to maintain, if possible, all the reference points in the investigation and, thus, to maximize the diversity.

\section{Selected case study}

For this work, the proposed optimization routine is implemented for both the IPM and IM and considers three criteria: the attainment of the targeted torque capability, $T_{\text {ref }}$ (Table I) as well as the maximization of both the motor's efficiency and torque density. Thus, the objective-function vector for both motor designs is defined as follows:

$$
F=\left[\begin{array}{lll}
w_{1} f_{1} & w_{2} f_{2} & w_{3} f_{3}
\end{array}\right]=\left[\begin{array}{lll}
\left|T_{\text {ref }}-T_{\mathrm{FEM}}\right| & \eta_{\mathrm{ref}}-\eta_{\mathrm{FEM}} & \frac{1}{\tau_{\mathrm{FEM}}}
\end{array}\right]
$$

considering weights $w_{1}, w_{2}, w_{3}$ equal to 1 for this analysis. In (1), $T_{\mathrm{FEM}}$ is the estimated average torque, $\eta_{\mathrm{FEM}}$ the estimated efficiency and $\tau_{\mathrm{FEM}}$ the estimated torque density obtained through time-stepping FE simulations of the aforementioned FE models. $T_{\mathrm{FEM}}$ is calculated according to Arkkio's method of numerical torque computation [15]. Arkkio's method was selected as it yields more accurate results compared to Maxwell's stress tensor formulation approach, by replacing the surface integral of the magnetic flux density over the motor's air-gap with a volume-averaging integral instead. Thereafter, $\tau_{\mathrm{FEM}}$ is determined by dividing $T_{\mathrm{FEM}}$ with the motor's active mass.

The estimated efficiency $\eta_{\text {FEM }}$ of each motor design is computed using the following expression:

$$
\eta_{\mathrm{FEM}}=\frac{T_{\mathrm{FEM}} \cdot \omega_{\mathrm{n}}}{T_{\mathrm{FEM}} \cdot \omega_{\mathrm{n}}+P_{\mathrm{Cu}}+P_{\mathrm{Fe}}}
$$

where $\omega_{\mathrm{n}}$ is the rotational nominal speed, $P_{\mathrm{Cu}}$ the copper losses dissipated in the stator winding (and the rotor bars for the case of IM) and $P_{\mathrm{Fe}}$ the iron losses in the steel laminations of stator and rotor cores. It should be noted that the $P_{\mathrm{Fe}}$ are calculated in post-processing using manufacturer's steel-measurement data and an analytical iron loss model considering static hysteresis losses and dynamic eddy current 
losses [16], [17]. Mechanical losses as well as power losses related to the modulation of the supply voltage are not included in the present study. In addition, in the case of the IPM, the induced eddy-current losses in the embedded magnets are considered negligible, assuming the use of permanent magnets with low electrical conductivity.

The selected decision variables of the optimization routine for both the IPM and IM design have been already introduced in the previous section (see Table III). In consequence, the optimization-variable vectors are expressed as:

$$
X_{\mathrm{IPM}}=\left[\begin{array}{lllllll}
\chi_{\text {split }} & \chi_{\text {tooth }} & W_{\text {mag }} & H_{\text {mag }} & \chi_{\text {aspect }}
\end{array}\right] \quad \& \quad X_{\mathrm{IM}}=\left[\begin{array}{lllll}
\chi_{\text {split }} & \chi_{\text {tooth }} & \chi_{\mathrm{w}, \text { bar }} & \chi_{\mathrm{h}, \text { bar }} & \chi_{\text {aspect }}
\end{array}\right]
$$

The variation of these selected variables within defined boundaries throughout the optimization procedure results in the determination of the non-dominated population members that comprise the desired Pareto-optimal set. The selection of the upper and lower limits for each of the decision variables was made by taking into account spatial and mechanical limitations.

\section{IPM cost-evaluation procedure}

As previously described, a certain number of new population members are produced in each generation during the optimization either though initialization, crossover or mutation. These individuals are compared based on their performance on the specified nominal operating point (Table I). This presupposes that they perform at maximum-torque-per-ampere (MTPA) operation at this point, while they are supplied with nominal voltage (calculated through the specified $V_{\mathrm{DC}}$ assuming space vector modulation control) and nominal current (related to the selected fixed stator current density, $J_{\mathrm{CU}}$ ). To achieve this for the IPM designs, a suitable cost-evaluation procedure was developed with the following steps:

Step 1: Analytical estimation of the optimal stator current angle, $\theta_{\mathrm{MTPA} \text {,analyt }}$.

In the case of an IPM motor, the produced electromagnetic torque can be estimated through the mathematical formula [18]:

$$
T_{\mathrm{e}}=\frac{3 p}{4}\left[\psi_{\mathrm{PM}} \hat{i}_{\mathrm{S}} \cos \theta+\frac{1}{2}\left(L_{\mathrm{q}}-L_{\mathrm{d}}\right) \hat{i}_{\mathrm{s}}^{2} \sin (2 \theta)\right]
$$

where $\hat{i}_{\mathrm{s}}$, in our case, is the nominal stator current amplitude, $\theta$ the stator current angle, $p$ the number of poles in the motor, $\psi_{\mathrm{PM}}$ the PM flux linkage and $L_{\mathrm{d}}$ and $L_{\mathrm{q}}$ the stator $\mathrm{d}$ - and q-axis inductances respectively, for the actual operating point. For a fixed $\hat{i}_{\mathrm{s}}$, the motor operates at MTPA when the produced torque is maximum. Thus, the corresponding optimal $\theta_{\mathrm{MTPA} \text {,analyt }}$ can be derived by equating the derivative of $T_{\mathrm{e}}$ with respect to $\theta$ to zero [18]:

$$
\theta_{\mathrm{MTPA}, \text { analyt }}=\sin ^{-1}\left(\frac{-\psi_{\mathrm{PM}}+\sqrt{\psi_{\mathrm{PM}^{2}+8\left(L_{\mathrm{q}}-L_{\mathrm{d}}\right)^{2} \hat{i}_{\mathrm{s}}^{2}}}}{4\left(L_{\mathrm{q}}-L_{\mathrm{d}}\right) \hat{i}_{\mathrm{s}}}\right)
$$

To obtain a time-efficient calculation of the above equation, the values of $\psi_{\mathrm{PM}}, L_{\mathrm{d}}$ and $L_{\mathrm{q}}$ are derived analytically. Specifically, the winding function is used to describe the spatial distribution of the stator magneto-motive force (MMF) in regards to the AC excitation of the IPM motor [19]. Moreover, due to the low relative permeability of the embedded magnets, an equivalent air-gap is considered, which varies with respect to the angle along the rotor circumference. In particular, for the angle-span in front of the $\mathrm{V}$-shaped magnets ( $\alpha_{\mathrm{p}}$ in Fig. 4), the effective air-gap is assumed to be $\delta+H_{\mathrm{mag}}$, while for the remaining pole-pitch, the air-gap is regarded as equal to $\delta$. The air-gap magnetic field distribution due to the stator current excitation can be obtained from the winding function and the air-gap inverse function, whereas the flux density due to the PM is calculated based on Ampere's law and flux conservation, accounting for the saturation in the rotor iron-bridges $(\approx 2 \mathrm{~T})$. Based on these computations, the quantities $\psi_{\mathrm{PM}}, L_{\mathrm{d}}$ and $L_{\mathrm{q}}$ can de derived [20]. 


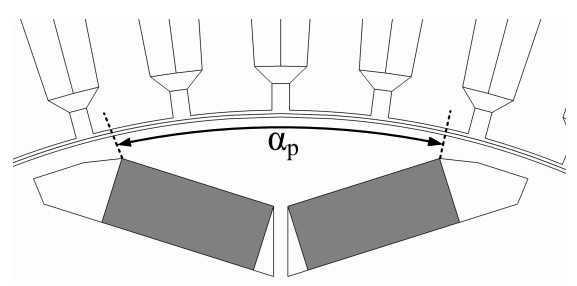

Fig. 4: Close-up of the angle-span $\alpha_{\mathrm{p}}$ in front of the PM in the rotor circumference.

Step 2: Numerical identification of the optimal stator current angle, $\theta_{\mathrm{MTPA}, \mathrm{FEM}}$.

The analytical estimation of the optimal stator current angle needs to be validated numerically. For this purpose, a number of static FEM simulations of each IPM individual are performed, where $\theta_{\text {FEM }}$ varies within a predefined search space ( $\max \pm 3.5 \mathrm{deg}$ ) in order to obtain numerically the highest torque capability. Starting the analysis with considering the center-angle equal to $\theta_{\mathrm{MTPA}}$,analyt, two additional FEM simulations of the investigated motor design are executed in each iteration, with assigned stator current angles higher and lower than the center-angle by a specified step-angle. The current angle that corresponds to the maximum $T_{\mathrm{FEM}}$ among the three FE models is identified and used as center-angle for the next iteration. The step-angle is set to 2 deg initially and is halved between successive iterations. The search is terminated with the acquisition of the desired $\theta_{\text {MTPA,FEM }}$ either when the step-angle becomes smaller than $0.5 \mathrm{deg}$ or the relative change in the maximum $T_{\mathrm{FEM}}$ is lower than $0.5 \%$ of the specified $T_{\text {target }}$.

Step 3: Satisfaction of the requirement of nominal voltage.

After computing the magnitude of the fundamental of the stator phase voltage though a transient FE analysis, the number of stator winding conductors, $n_{\mathrm{s}}$, is re-adjusted in order to achieve the specified voltage value. Finally, the objectives of $T_{\mathrm{FEM}}, \tau_{\mathrm{FEM}}$ and $\eta_{\mathrm{FEM}}$ can be determined through a final FE simulation.

The overall procedure is presented in the concise flowchart of Fig. 5a.

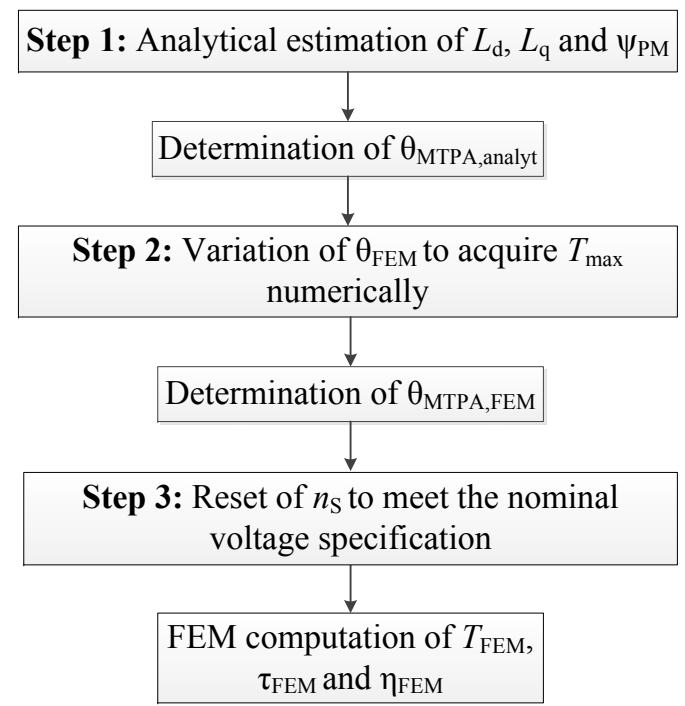

(a)

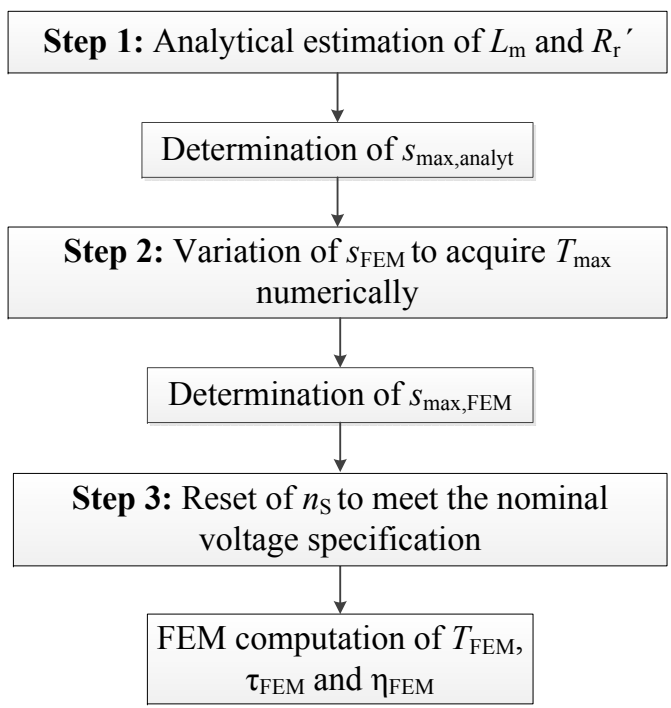

(b)

Fig. 5: Flowcharts of the (a) IPM and (b) IM cost-evaluation procedures.

\section{IM cost-evaluation procedure}

Regarding the IM designs, a similar cost-evaluation routine as in the case of IPM was followed in order to calculate the required objectives for each individual at MTPA operation. However, in this topology, the determining factor for identifying the MTPA operation is the slip of the motor. 
Step 1: Analytical estimation of the optimal slip of the motor, $s_{\max , \text { analyt }}$.

In an IM, the produced electromagnetic torque, $T_{\mathrm{e}}$, can be expressed through the angular slip-frequency of the rotor, $\omega_{\text {slip }}$, as follows:

$$
\omega_{\text {slip }}=s \frac{\pi n_{\mathrm{n}}}{30(1-s)} \quad, \quad T_{\mathrm{e}}=\frac{3 p}{4} L_{\mathrm{M}} \hat{i}_{\mathrm{s}}^{2} \frac{\frac{L_{\mathrm{M}} \omega_{\mathrm{slip}}}{R_{\mathrm{r}}^{\prime}}}{1+\left(\frac{L_{\mathrm{M}} \omega_{\mathrm{slip}}}{R_{\mathrm{r}}^{\prime}}\right)^{2}}
$$

where $s$ is the slip, $p$ the number of poles in the motor, $L_{\mathrm{M}}$ the equivalent total magnetizing inductance and $R_{\mathrm{r}}^{\prime}$ the rotor resistance referred to the stator side in the steady-state equivalent circuit of the IM model [21],[22]. For fixed $\hat{i}_{\mathrm{s}}$, MTPA operation is achieved when the produced torque capability of the IM is maximized. The slip of the motor at that instant, $s_{\max \text {,analyt }}$, can be estimated by letting the derivative of $T_{\mathrm{e}}$ with respect to $s$ to zero.

Step 2: Numerical identification of the optimal slip of the motor, $s_{\max , \text { FEM }}$.

For the validation of the analytical estimation, a similar process as in the IPM is followed: a number of time-harmonic analysis FEM simulations for each population member are performed, where, this time, $s_{\mathrm{FEM}}$ is varied in order to compute numerically the maximum output torque. The step-slip is set initially to 0.005 and is halved between successive iterations. The stopping criteria in order to identify the required $s_{\text {max,FEM }}$ are met either when the step-slip becomes smaller than 0.001 or the relative change in the maximum $T_{\mathrm{FEM}}$ is lower than $0.5 \%$ of the specified $T_{\text {target }}$.

Step 3: Satisfaction of the requirement of nominal voltage.

The phase voltage in the stator winding is calculated via a transient FE analysis, which uses the magneticfield solution of a time-harmonic FE model as initial condition in order to reach steady-state faster. Afterwards, $n_{\mathrm{s}}$ is re-set to meet the voltage specification and the required objectives (referring to $T_{\mathrm{FEM}}$, $\tau_{\mathrm{FEM}}$ and $\eta_{\mathrm{FEM}}$ ) are evaluated in a subsequent transient FE analysis.

The above procedure is illustrated in the form of a flowchart in Fig. $5 b$.

\section{Results and discussion}

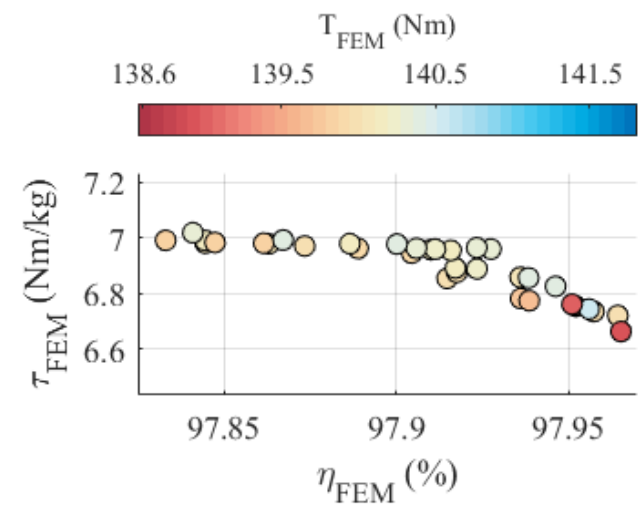

(a)

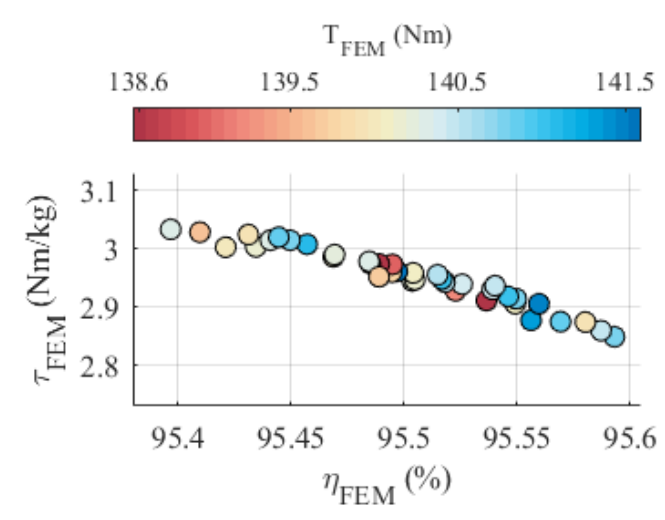

(b)

Fig. 6: 2-D projections of the (a) IPM and (b) IM Pareto front.

Both IPM and IM optimization problems consist of $D=5$ decision variables and $m=3$ objectives. Therefore, their Pareto-optimal solutions lie on a three-dimensional (3-D) search space. For this study case, the parameters of the optimization algorithms were set as follows: population size $N=40$, number of generations $N_{G}=25$, crossover probability $p_{c}=0.9$ and mutation probability $p_{m}=1 / D$. The resulting Pareto-optimal solutions are presented in Fig. 6. 
As it can de observed in Fig. 6, the optimal designs of both motor topologies meet successfully the requirement of the aimed torque capability, $T_{r e f}$. In addition, the conflicting nature of the two objectives of torque density, $\tau_{F E M}$ and efficiency, $\eta_{F E M}$ is clearly illustrated. Finally, it can be seen that the optimization routine for the nominal operating point results in optimal IM designs with lower efficiency and inherently lower torque density values than the respective IPM designs.

\section{Conclusion}

This paper presents the design process of an IPM synchronous motor and an induction motor using an evolutionary many-objective algorithm. Both topologies have been optimized considering three conflicting objectives: torque capability, efficiency and torque density. It is shown that the obtained Paretooptimal solutions of both topologies satisfy the required criteria, rendering the proposed many-objective approach an effective optimization tool that offers feasible compromises among various targets. Additional investigation of thermal and mechanical aspects is needed in order to further support the suitability of both motor topologies for the specific application.

\section{References}

[1] I. Boldea et al.: Automotive Electric Propulsion Systems With Reduced or No Permanent Magnets: An Overview, IEEE Transactions on Industrial Electronics, vol. 61, no. 10, pp. 5696-5711, Oct. 2014.

[2] N. Hashemnia and B. Asaei: Comparative study of using different electric motors in the electric vehicles, 2008 18th International Conference on Electrical Machines, Vilamoura, 2008, pp. 1-5.

[3] R. Cao et al.: Quantitative Comparison of Flux-Switching Permanent-Magnet Motors With Interior Permanent Magnet Motor for EV, HEV, and PHEV Applications, IEEE Transactions on Magnetics, vol. 48, no. 8, pp. 2374-2384, Aug. 2012.

[4] M. Kimiabeigi et al.: High-Performance Low-Cost Electric Motor for Electric Vehicles Using Ferrite Magnets, IEEE Transactions on Industrial Electronics, vol. 63, no. 1, pp. 113-122, Jan. 2016.

[5] M. Zeraoulia et al.: Electric Motor Drive Selection Issues for HEV Propulsion Systems: A Comparative Study, IEEE Transactions on Vehicular Technology, vol. 55, no. 6, pp. 1756-1764, Nov. 2006.

[6] J. Goss et al.: A comparison of an interior permanent magnet and copper rotor induction motor in a hybrid electric vehicle application, 2013 International Electric Machines \& Drives Conference, Chicago, IL, 2013, pp. 220-225.

[7] C. T. Krasopoulos et al: Multicriteria PM Motor Design Based on ANFIS Evaluation of EV Driving Cycle Efficiency, IEEE Transactions on Transportation Electrification, vol. 4, no. 2, pp. 525-535, June 2018.

[8] A. G. Sarigiannidis et al: Fast Adaptive Evolutionary PM Traction Motor Optimization Based on Electric Vehicle Drive Cycle, IEEE Transactions on Vehicular Technology, vol. 66, no. 7, pp. 5762-5774, July 2017.

[9] K. Deb: Multi-objective optimization using evolutionary algorithms, Chichester, UK, Wiley, 2001.

[10] K. Deb et al.: A fast and elitist multiobjective genetic algorithm: NSGA-II, IEEE Transactions on Evolutionary Computation, vol. 6, no. 2, pp. 182-197, Apr. 2002.

[11] J. Le Besnerais et al.: Multiobjective optimization of induction machines including mixed variables and noise minimization, IEEE Transactions on Magnetics, vol. 44, no. 6, pp. 1102-1105, June 2008.

[12] J. Maitre et al.: An effective identification of the induction machine parameters using a classic genetic algorithm, NSGA II and $\theta$-NSGA III, 2015 6th International Conference on Information, Intelligence, Systems and Applications (IISA), pp. 1-6, July 2015.

[13] K. Deb and H. Jain: An Evolutionary Many-Objective Optimization Algorithm Using Reference-PointBased Nondominated Sorting Approach, Part I: Solving Problems With Box Constraints, IEEE Transactions on Evolutionary Computation, pp. 577-601, Aug. 2014.

[14] T. A. Burress et al.: Evaluation of the 2010 Toyota Prius hybrid synergy drive system, Oak Ridge National Lab.(ORNL), Oak Ridge, TN (United States),ORNL/TM-2010/253, Mar. 2011.

[15] A. Arkkio: Analysis of induction motors based on the numerical solution of the magnetic field and circuit equations, Acta Polytechnica Scandinavica, Electrical Engineering, vol 59, Helsinki University of Technology, 1987.

[16] H. Jordan: Die ferromagnetischen Konstanten für schwache Wechselfelder, Elektr. Nach. Techn., vol. 1, p. 8, 1924.

[17] A. Krings and J. Soulard: Overview and comparison of iron loss models for electrical machines, Journal of Electrical Engineering, vol. 10, no. 3, pp. 162-169, 2010.

[18] G. Rang et al.: A MTPA control scheme for an IPM synchronous motor considering magnet flux variation caused by temperature, Nineteenth Annual IEEE Applied Power Electronics Conference and Exposition, pp. 1617-1621, vol.3, 2004. 
[19] J. C. Moreira and T. A. Lipo: Modeling of saturated ac machines including air gap flux harmonic components, IEEE Trans. Ind. Appl.,vol. 28, no. 2, pp. 343-349, Mar./Apr. 1992

[20] T. A. Lipo: Introduction to AC Machine Design, Univ. Wisconsin, Madison, WI, 2004.

[21] O. Wallmark, AC Machine Analysis - Fundamental Theory, Stockholm, Sweden: KTH Royal Institute of Technology, 2018.

[22] P.Vas, Sensorless vector and direct torque control, (Monographs in electrical and electronic engineering 42). Oxford: Oxford Univ. Press, 1998. 